The challenges of multi-professional work most emphasised were lack of time, personnel turnover and communication issues. Research findings on the views of the various actors were supported by the interviews with the children and young people. These interviews were taken into consideration in the development of the model. Research finding obtained from the third research phase will be presented at the Safety 2016 World Conference.

Conclusions When attending to the concerns of children and young people, account must be taken of the views expressed by those who need help as well as by those parties that are engaged in collaboration.

\section{RISK FACTORS OF TRAUMATIC BRAIN INJURIES BETWEEN THE YOUNGSTERS}

Enkelejda Shkurti, Diamant Shtiza. University of Medicine, Albania

10.1136/injuryprev-2016-042156.930

Background The elevated occurrence of traumatic brain injuries (TBI) between the youngsters has brought much spotlight to this area in current years. Sports harms have been recognised as a major instrument. Even though vigour drinks, counting those mixed with alcohol, are frequently used by juvenile athletes and other youngsters they have not been observed in relation to TBI. We examined the occurrence of youngsters' TBI and its alliances with vigour drinks, alcohol and energy drink assorted in with alcohol use.

Methods Data were obtained from the Institute of Public Health in Albania. This cross-sectional school study comprised 7430 students from high school (aged 14-20) who fulfilled self-administered questionnaires in classrooms.

Results Between the participants, 25.6\% (95\% CI: 21.3, 23.8) expressed a record of TBI. Sports harms remain the major instrument of a current TBI $(44.8 \%$, 95\% CI: 40.6, 49.4). Logistic regression demonstrated that compared with youngsters who never maintained a TBI, the odds of supporting a current TBI were bigger than those using alcohol, vigour drinks, and energy drinks assorted in with alcohol than abstainers. In relation to current TBI thanks to other reasons of harm, youngsters who carried on a current TBI whereas playing sports had bigger odds of current energy drinks use than abstainers.

Conclusions TBI continues to be a immobilising and ordinary circumstance abetween the youngsters and the use of alcohol, vigour drinks, and alcohol assorted with vigour drinks promote the enhancement of the odds of TBI between the youngsters. These alliances necessitate additional research.

\section{IMPLEMENTING SAFETY CULTURE IN TEACHER EDUCATION}

${ }^{1}$ Eila Lindfors, ${ }^{2}$ Brita Somerkoski. 'University of Turku, Teacher Education, Rauma Unit, Finland; ${ }^{2}$ University of Turku, Teacher Education, Rauma Unit

\subsection{6/injuryprev-2016-042156.931}

Background During the past few years the incidents of extreme violence and the increase of unintentional injuries and accidents have created situations where new safety procedures are needed. Safe learning environment should be a safe working environment for teachers. According to the target programs and visions, safety and wellbeing can be seen as basic values of the society.
Objective The New National core Curriculum contains broadbased competence issues such as "Looking after oneself, managing daily activities and safety". The renewed curricula also includes practical safety issues such as knowing the basic safety signs and symbols, conducting appropriate behaviour in traffic and avoiding dangerous situations. The renewed curriculum sets new challenges also for teacher education. There is a need for new teacher's safety competence with knowledge and attitude but also the practical skills.

Results The curricula for the University of Turku, teacher education in Rauma unit were renewed. The new teacher education curricula include safety and security issues such as safety competence and school subject didactical safety as well as safety in practical training. As a part of their studies the prospective teachers participate to educational lessons or debates, safety walks, firedrills and monitoring of unintentional injuries. This is preparing the teachers to act safely when working at the school.

In addition The Developing Network of Safety in Schools (OPTUKE) has been established. The aim was to advance a wide, systematic and open safety culture in schools as well as to support the process of making safety culture more visible with the help of a pedagogic point of view on safety.

Conclusions These measures strengthen prospective teachers' safety competence, resilience and ability to respond in the emergency situations

\section{PREVENTING SOCIAL EXCLUSION WITH VOLUNTARY FIRE DEPARTMENT TRAINING ACTIVITIES}

${ }^{1}$ Brita Somerkoski, ${ }^{2}$ Terhi Kivijärvi, ${ }^{3}$ Virpi Hurula. 'National Institute for Health and Welfare

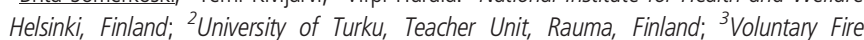
Brigade of Vahto, Vahto, Finland

10.1136/injuryprev-2016-042156.932

Preventing social exclusion with voluntary fire department training activities

Brita Somerkoski, Planning Officer, National Institute of Health and Welfare

Background Social exclusion among youth is one of the major social problems in Finland. Therefore it is seen as the biggest threat to internal security. A low level of education, a lack of hobbies and a lack of sufficient skills increase the risk of becoming excluded from the labour market and from society. Moreover, inherited problems, such as transgenerational social exclusion, are seen as relatively new and worrying phenomena in Finland.

Objectives The Yli Hyvä Juttu -program is an early intervention, where at-risk children and young people are directed to participate in interesting free-time activities at a local voluntary fire department. This is done in collaboration with the local authorities, such as social, youth and educational services. The target groups are children and young people between the ages of 7 and 17 , especially those who have been antisocial, lonely or in need of boundaries. A study of the possibilities of the program was carried out among the youth trainers and social workers. The presentation will show the forms of activities as well as the results of the latest outcome study of the possibilities of Yli Hyvä Juttu working model.

Results Through the voluntary fire department's training activities, children and young people learn firefighter skills and other safety activities. Youth trainers in fire brigades are educated to meet the possible special needs of young persons. Also group dynamics such as team control and team spirit are taught to the trainers. 
Conclusions Voluntary fire brigade gives hope, new goals and empowerment to the vulnerable group of young people.

\section{IMPACT OF FIRST AID TRAINING ON THE FIRST AID KNOWLEDGE AND SKILLS CAPACITY OF PRIMARY SCHOOL TEACHERS IN IBADAN, NIGERIA}

${ }^{1}$ Abdulmumin Ibrahim, ${ }^{2}$ Nadia Sam-Agudu, ${ }^{1}$ Ugbede Omave, ${ }^{1}$ Boniface Ushie ${ }^{1}$ Adesola Olumide, ${ }^{1}$ Olayemi Omotade. ${ }^{1}$ University of Ibadan, Nigeria; 2 Institute of Human Virology Nigeria, Nigeria

\subsection{6/injuryprev-2016-042156.933}

Background Acute illness and injury are major causes of child morbidity and mortality. Young children are prone to injury, and schools in developing countries are often not optimally childproofed. In Nigeria, primary schoolteachers supervise students in an often injury-prone environment, with poor access to emergency services. First Aid (FA) can make a significant difference in outcome for a young child injured at school. This study was conducted to assess FA knowledge and skills in a cohort of primary school teachers and evaluate the effect of a training program on the cohort's FA capacity.

Methods We randomly selected 151 teachers from 16 primary schools in Ibadan. A 26-point survey and simulated scenarios graded on an 18-point scale assessed FA knowledge and skills, respectively. Based on identified gaps, a training program was developed and applied to a randomly selected subset of 70 of the 151 teachers, assigning them to intervention $(\mathrm{N}=36)$ and control $(\mathrm{N}=34)$. Controls were given an HIV education talk. FA knowledge and skills were measured immediately and 3 months post-intervention. FA knowledge was rated poor $(<13)$, fair $(13-$ $17)$ and good (>17); skills were rated poor $(<9)$, fair $(9-11)$ and good (>11). Chi-square, t-test and ANOVA were used to compare means and to test for associations.

Results Respondents' mean age was $41.44 \pm 9.90$ years, and 95.4\% had poor baseline knowledge. There was no difference in mean FA knowledge between intervention $(7.69 \pm 1.97)$ and control $(7.29 \pm 2.47)$ at baseline $(p=0.49)$. Mean baseline FA skills scores between intervention $(0.5 \pm 2.27)$ and control $(0.65 \pm 1.28)$ were similar $(\mathrm{p}=0.59)$. Compared to baseline, there was a significant increase in mean FA knowledge immediately $(20.83 \pm 1.00, \mathrm{p}<0.001)$ and 3 months post intervention $(18.24 \pm 2.00, \mathrm{p}<0.001)$. Mean FA skills scores also improved from baseline, immediately $(12.72 \pm 1.80, \mathrm{p}<0.001)$ and 3 months post intervention $(9.64 \pm 2.03, \mathrm{p}<0.001)$. There were no significant changes in FA knowledge or skills in the control group.

Conclusions First aid knowledge and skills among primary school teachers in Ibadan was poor. The training program led to a significant and sustained improvement in teachers' FA capacity. Primary school teachers can be trained and retrained to provide appropriate and timely basic care for students injured at school.

\section{INTERVENTIONS TO REDUCE INJURIES AMONG STUDENTS IN PRIMARY AND SECONDARY SCHOOLS IN XINING CITY}

Min-Ru Zhou, Su-Xia Zhou, Xu Zhi-Hua, Sha Qiong-Yue. QinHai Centre for Disease Prevention and Control, Qing Hai, China

10.1136/injuryprev-2016-042156.934
Background Injuries are a global public health problem, and child injuries are particularly outstanding considering that children are free from most other health problems. We carried out an investigation on injuries in three primary and two secondary schools in Xining in 2003, and the results showed that the incidence of injuries was as high as $26.24 \%$. Interventions were taken to reduce injuries in one primary and one secondary schools in Xining from 2006 to 2008.

Methods Baseline survey was carried out before intervention. School leaders, teachers, students, doctors and CDC experts were invited to devise intervention measures and discuss the implementation approaches. Between 2006 and 2008, several interventions were applied: dispensing injury prevention brochures; composing injury prevention knowledge into songs and printing them on school timetable; making posters of fall prevention; compiling the proposal of creating a safe and peaceful school environment and printing related contents on the outer packing of ballpoint pen; dispensing accidental childhood injury prevention handbook named "love, with safety first"; and sponsoring a drawing contest with the theme of injury prevention. Besides these measures, we also heightened and strengthened stair railings, placed warning signs on accident-prone locations, and improved school security.

Results The results of baseline survey showed that student knowledge awareness rate of fall injury prevention was $63.76 \%$; the overall incidence of injuries in students in one year was 21.51\%; and the leading types of injuries were fall (8.69\%), collision (3.85\%), traffic accident (2.14\%), burn (1.71\%), cut $(1.28 \%)$ and animal bites $(1.28 \%)$ according to the injury frequency. After intervention, the awareness rate of fall injury prevention in students increased to $98.86 \%$; the overall incidence of injuries decreased to $18.06 \%$; and the incidences of fall, collision, traffic accident, burn, cut and animal bites changed to $8.19 \%$, $3.03 \%, 1.82 \%, 1.52 \%, 1.37 \%$ and $0.91 \%$, respectively.

Conclusions Interventions could effectively reduce injuries among students. Our work laid a foundation for further interventions on child injuries in Xining city.

\section{SIGNIFICANCE OF TRADITIONAL MASCULINITY FOR THE PREDICTION OF INJURIES IN MALE ADOLESCENTS}

Natko Geres, Milan Milosevic, Aida Mujkić. University of Zagreb, School of Medicine, Andrija Stampar School of Public Health, Zagreb, Croatia

\subsection{6/injuryprev-2016-042156.935}

Background Risk from injuries and accidents is increased for men in comparison to women. Young men are especially at risk. There is a complexity of interactions between environmental and personal factors (masculinity, impulsivity, depression and indicators of socioeconomic status, micro and macro environment) in relation to risky behaviours and injuries and accidents. Men have higher incidence of risky behaviours (for instance, alcohol use) that contribute to injuries. Existing studies put emphasis on exploring traditional masculine attitudes as factors contributing to this trend.

Methods Research was implemented by using already existing, validated tools on 1. Injuries and accidents: Youth Risk Behaviour Survey-YRBS (CDC, 2013), 2. Masculinity: Male Role Norm Inventory- Adolescent- revised (MRNI-A-R), 3. Socioeconomic status: Indicators of socioeconomic status for adolescents using Family affluence scale as measure of family wealth and 4. Experiences of injuries and accidents using Injury Checklist )Jelalian, 